\title{
Article \\ Modeling and Spatial Diversity-Based Receiving Improvement of In-Flight UAV FSO Communication Links
}

\author{
Anrui Zheng ${ }^{1,2}$, Yang Huang ${ }^{1,2}$ and Shiming Gao ${ }^{1,2, *}$ \\ 1 Centre for Optical and Electromagnetic Research, State Key Laboratory of Modern Optical Instrumentation, \\ International Research Centre for Advanced Photonics, Zhejiang University, Hangzhou 310058, China; \\ Zhenganrui@zju.edu.cn (A.Z.); 21930002@zju.edu.cn (Y.H.) \\ 2 Ningbo Research Institute, Zhejiang University, Ningbo 315100, China \\ * Correspondence: gaosm@zju.edu.cn
}

check for updates

Citation: Zheng, A.; Huang, Y.; Gao, S. Modeling and Spatial

Diversity-Based Receiving Improvement of In-Flight UAV FSO Communication Links. Appl. Sci. 2021, 11, 6365. https://doi.org/ 10.3390/app11146365

Academic Editor: Alberto Gatto

Received: 1 June 2021

Accepted: 6 July 2021

Published: 9 July 2021

Publisher's Note: MDPI stays neutral with regard to jurisdictional claims in published maps and institutional affiliations.

Copyright: (c) 2021 by the authors. Licensee MDPI, Basel, Switzerland. This article is an open access article distributed under the terms and conditions of the Creative Commons Attribution (CC BY) license (https:// creativecommons.org/licenses/by/ $4.0 /)$.

\begin{abstract}
An in-flight unmanned aerial vehicle (UAV) free-space optical (FSO) communication channel model is proposed by considering the beam deviation of the UAV under different motion states and the phase distortion caused by atmospheric turbulences. The influence of the different motion states and turbulences on the communication quality is evaluated through phase screen and Monte Carlo methods. When the average bit error rate (BER) is $10^{-5}$, the signal-to-noise ratio (SNR) should be increased from $13 \mathrm{~dB}$ to $20 \mathrm{~dB}$ when the tilt angle of the UAV increases from 0 to $5 \mathrm{mrad}$. An SNR of up to $20 \mathrm{~dB}$ is required when the variance of the wind $\sigma_{\alpha}^{2}$ is $2 \mathrm{mrad}$. The performance of the in-flight UAV FSO link can be effectively improved through spatial diversity receiving technology. The BER of lower than $10^{-5}$ can be obtained just with an SNR of $13 \mathrm{~dB}$ if the spatial diversity array with four receivers is used.
\end{abstract}

Keywords: in-flight; unmanned aerial vehicles; free-space optical communication; tilt angle; wind resistance; spatial diversity

\section{Introduction}

Free-space optical (FSO) communication technology has been widely used in commercial and military fields due to its advantages such as large transmission capacity, rich spectrum resource, good confidentiality, and good directivity [1-3]. Moreover, with the use of unmanned aerial vehicles (UAVs) gradually shifting from the military field to the commercial and civil fields, high-speed data transmission is often needed in some special working environments, such as terrain survey and disaster detection. For the above reasons, FSO communication technology is naturally introduced into the field of UAV, so as to establish high-speed optical communication networks in ground-to-ground and air-to-air scenarios.

The FSO communication link between UAVs is fundamental to improve the coverage range and networking flexibility of UAVs. In recent years, UAVs and high-altitude platforms are often used as relays to support wireless long-range links between two terrestrial stations [4,5], and the channel modeling of UAV FSO communication has also been fully discussed [6-8]. In consideration of turbulence intensity, field angle, spatial position, pointing deviation, and transmitting power, a closed-form statistical channel model, which is a mathematical statistical channel model using a finite number of standard operations of the UAV communication has been proposed, and the above parameters have been optimized to improve the communication quality $[9,10]$. At the same time, a removable lens array has been used to change the beam divergence mechanism of the transmitter to enhance the stability of the link [7].

However, previous studies were only limited to the communication between hovering UAVs without considering the influence of flying and wind resistance. In hovering states, the UAV FSO communication will only be affected by the jitter and angle deviation [9-11]. 
Taking the star formation UAV group as instance, the central UAV is in the UAV hovering state, and the rest of the UAVs fly on the same level against the central UAV [12]. During the flight mission, the central UAV sends information to the other UAVs through FSO communications while the tilt of UAV bodies will cause the light beam to be non-orthogonal to the lens at the receiving end. The wind resistance is also one of the main causes of UAV's jitters. Therefore, an FSO communication link model between in-flight UAVs that includes all the jitter, deviation, receiving error, and wind resistance effects is essential to be established.

The spatial diversity technology can greatly increase the redundancy of the system by receiving the incoherent signals at different locations and improve the stability of the link. A receiver array with multiple isometric receivers, which combines multiple groups of mutually independent fading signals at the receiving end is utilized in this technology, and a strong anti-interference effect on the beam offset and light intensity fluctuation exists [13]. Equal gain combining (EGC) is a common linear combination algorithm of combining schemes of diversity technology, which can set the weight coefficient of the signals received in each road as 1, and merge them into one road signal to achieve signal gain [13-15]. In this way, the sensitivity of the FSO link can be effectively improved.

In this paper, a new FSO communication channel model for the in-flight receiving UAV is established. In consideration of the atmospheric turbulence and the tilt and jitter caused by the UAV flight, the bit error rate (BER) and the outage probability are simulated versus the signal-to-noise ratio (SNR), and the effect of spatial diversity technology on communication quality improvement is studied. Moreover, the improvement of communication quality by spatial diversity technology is analyzed.

\section{Theoretical Model of the In-Flight UAV FSO Communication Link}

When the UAV at the receiving end is flying horizontally, the arrival angle between the centerline of the beam and the receiving plane is not only related to the pointing deviation caused by the jitter of the receiving end, but also needs to consider the tilt angle of the receiving plane.

In a wireless optical communication link, the received signal at the receiving end can be expressed as [11]:

$$
y=h x+n
$$

where $x$ is the signal emitted at the transmitting end and $n$ is the additive white Gaussian noise (AWGN). $h$ is the channel coefficient that can be given by the following equation:

$$
h=h_{t} h_{a} h_{g}
$$

In Equation (2), $h_{t}$ is the attenuation and turbulence coefficient of the atmospheric channel; $h_{a}$ represents the link interrupt coefficient, which indicates whether the spot is detected on the detection plane; and $h_{g}$ is the geometric and pointing error coefficient, which represents the position deviation between the spot center and the lens center at the receiving end.

As for the attenuation of the optical signal by the influence of atmospheric turbulence, the distribution of $h_{t}$ can be described by the Gamma-Gamma model [16]:

$$
f\left(h_{t}\right)=\frac{2(\alpha \beta)^{\frac{(\alpha+\beta)}{2}}}{\Gamma(\alpha) \Gamma(\beta)} h_{t}\left[\frac{(\alpha+\beta)}{2}\right]-1 K_{\alpha-\beta}\left(2 \sqrt{\alpha \beta h_{t}}\right)
$$

where $K_{n}(\cdot)$ is the $n$-th order modified Bessel function of the second kind, $\Gamma(\cdot)$ is the Gamma function, $\alpha$ and $\beta$ represent the effective number of the large-scale and small-scale eddies, respectively: 


$$
\begin{aligned}
& \alpha=\left\{\exp \left[\frac{0.49 \sigma_{R}^{2}}{\left(1+1.11 \sigma_{R}^{\frac{12}{5}}\right)^{\frac{7}{6}}}\right]-1\right\}^{-1} \\
& \beta=\left\{\exp \left[\frac{0.51 \sigma_{R}^{2}}{\left(1+0.69 \sigma_{R}^{\frac{12}{5}}\right)^{\frac{5}{6}}}\right]-1\right\}^{-1}
\end{aligned}
$$

where the Rytov variance is expressed as:

$$
\sigma_{R}^{2}=1.23 k^{\frac{7}{6}} C_{n}^{2} L^{\frac{11}{6}}
$$

In Equation (3), $k$ is the wave number, $C_{n}^{2}$ is the atmospheric refractive index structural parameter, and $L$ is the propagation distance. In this work, the phase screen method is used to simulate the atmospheric turbulence in the Gamma-Gamma model [17-19].

When a multi-rotor UAV is flying in a straight and horizontal flight, a small tilt will exist between the fuselage and the $y$ axis, as shown in Figure 1. The size of the tilt angle is related to the speed of the UAV, which affects the link performance through the coefficient $h_{a}$. As shown in Figure 2a, assuming the UAV at the receiving end is flying horizontally, the included angle between the central axis and the $y$ axis is denoted as $\varphi$. Since the plane of the lens at the receiving end is parallel to the central axis of the UAV, the angle between the plane of the lens and the $y$ axis is also $\varphi$. The angle between the centerline of the beam and the perpendicular direction of the lens plane is defined as the arrival angle $\theta_{a}$, and the pointing deviation caused by the jitter inherent in the receiving and transmitting end is defined as $\theta$.

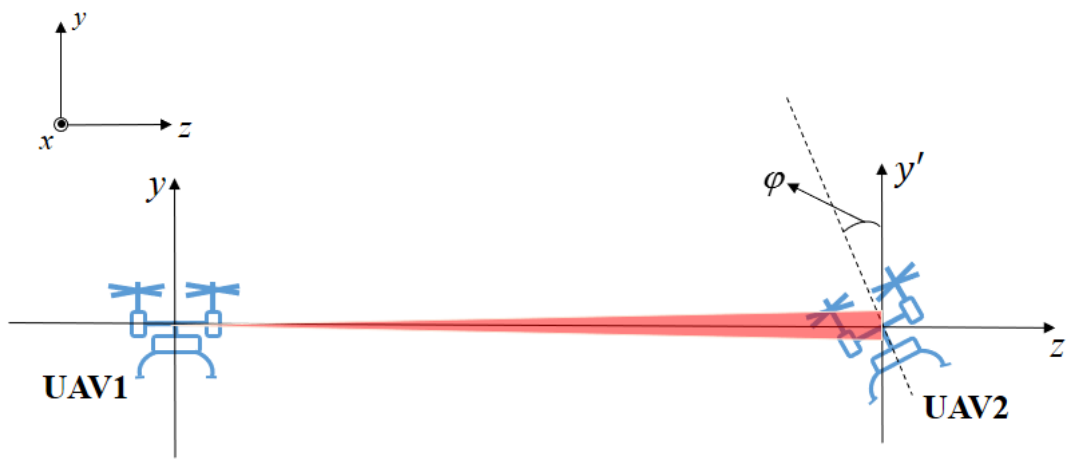

Figure 1. Diagram of the in-flight unmanned aerial vehicle (UAV) free-space optical (FSO) communication.

Here, the UAV is assumed to fly at the same altitude, and it is reasonable to choose a case without loss of generality to describe the relationship between $\theta_{a}$ and $\varphi$. As shown in Figure $2 \mathrm{~b}$, the direction of the light beam is parallel to the $y-z$ plane, and the angle between the beam axis and the $z$ axis is $\theta$. According to the diffraction theory, the communication link will be interrupted when the main lobe of the Airy disk is outside of the detection region of the detector. In the state of horizontal flight, $\theta_{a}$ can be expressed as $\theta_{a}=\theta-\varphi$. Therefore, the link will be interrupted when $\theta_{a}$ is larger than the receiver's field angle $\theta_{F O V}$. The probability density function (PDF) of $\theta_{a}$ is given by [10]:

$$
f\left(\theta_{a}\right)=\frac{|\theta-\varphi|}{\sigma_{r_{0}}^{2}+\sigma_{t_{0}}^{2}} \exp \left(-\frac{(\theta-\varphi)^{2}}{2\left(\sigma_{r_{0}}^{2}+\sigma_{t_{0}}^{2}\right)}\right)
$$


where $\sigma_{t o}^{2}$ and $\sigma_{r o}^{2}$ are the variances of the angle deviation of the transmitter and the receiver respectively. $\theta$ can be expressed as:

$$
\theta=\sqrt{\left(\theta_{t x}+\theta_{r x}\right)^{2}+\left(\theta_{t y}+\theta_{r y}\right)^{2}}
$$

where $\theta_{t x}$ and $\theta_{r x}$ represent the angle deviations of the transmitting end and the receiving end in the $x$ direction respectively, $\theta_{t y}$ and $\theta_{r y}$ represent the angle deviations of the transmitting end and the receiving end in the $y$ direction, respectively.
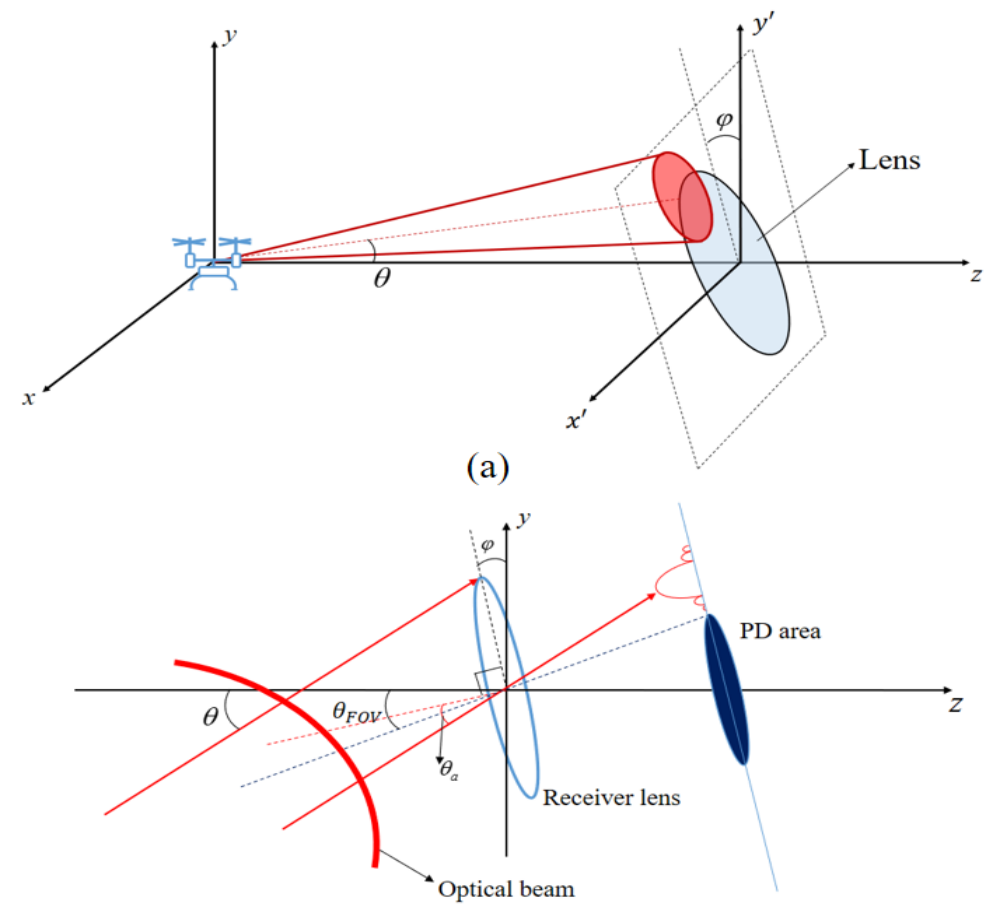

(b)

Figure 2. Schematic diagram of the receiving plane at the receiving end UAV. (a) 3D schematic diagram; (b) $y$-z plane diagram.

According to the above discussion, $h_{a}$ has two possible values. The communication will be interrupted when $\theta_{a}$ is greater than $\theta_{F O V}$, i.e., $h_{a}=0$; the communication interruption will not be induced when $\theta_{a}$ is less than or equal to $\theta_{F O V}$, i.e., $h_{a}=1$. The value condition of $h_{a}$ is given by the following equation:

$$
h_{a}=\left\{\begin{array}{c}
0 \theta>\theta_{F O V}-\varphi \text { or } \theta_{a}<-\left(\theta_{F O V}+\varphi\right) \\
1 \theta \leq \theta_{F O V}-\varphi \text { and } \theta_{a} \geq-\left(\theta_{F O V}+\varphi\right)
\end{array}\right.
$$

Therefore, the probability density function (PDF) of $h_{a}$ is given by:

$$
\begin{aligned}
& f\left(h_{a}\right)=\left[\frac{1}{2} \exp \left(-\frac{\left(\theta_{F O V}-\varphi\right)^{2}}{2\left(\sigma_{r_{0}}^{2}+\sigma_{t_{0}}^{2}\right)}\right)+\frac{1}{2} \exp \left(-\frac{\left(\theta_{F O V}+\varphi\right)^{2}}{2\left(\sigma_{r_{0}}^{2}+\sigma_{t_{0}}^{2}\right)}\right)\right] \delta\left(h_{a}\right) \\
& +\left\{1-\left[\frac{1}{2} \exp \left(-\frac{\left(\theta_{F O V}-\varphi\right)^{2}}{2\left(\sigma_{r_{0}}^{2}+\sigma_{t_{0}}^{2}\right)}\right)+\frac{1}{2} \exp \left(-\frac{\left(\theta_{F O V}+\varphi\right)^{2}}{2\left(\sigma_{r_{0}}^{2}+\sigma_{t_{0}}^{2}\right)}\right)\right]\right\} \delta\left(h_{a}-1\right)
\end{aligned}
$$

where $\delta(\cdot)$ is the Dirac function.

In the hovering UAV optical communication, the position deviation of the transceiver $\mathrm{UAV}$, and the angle deviation of the transmitter UAV are the main factors that cause the deviation of the center of the spot and the center of the lens. The geometric and pointing error coefficient $h_{g}$ is defined as the proportion of the light intensity of the coincidental part 
of the spot and the lens to the total light intensity. Here we assume that the transmitting $\mathrm{UAV}$ is hovering while the receiving UAV is yawing at the same altitude. In this case, the UAV at the receiving end will be affected by the random lateral wind resistance in the horizontal direction, which will cause the fuselage to wobble around the $y$ axis, thus causing the lens plane to be no longer orthogonal to the centerline of the beam. As shown in Figure 3, the projection of the lens on the $x-y$ plane becomes an ellipse instead of a circle. At this point, the intensity of the part where the lens and spot coincide is different from that in the hovering state.

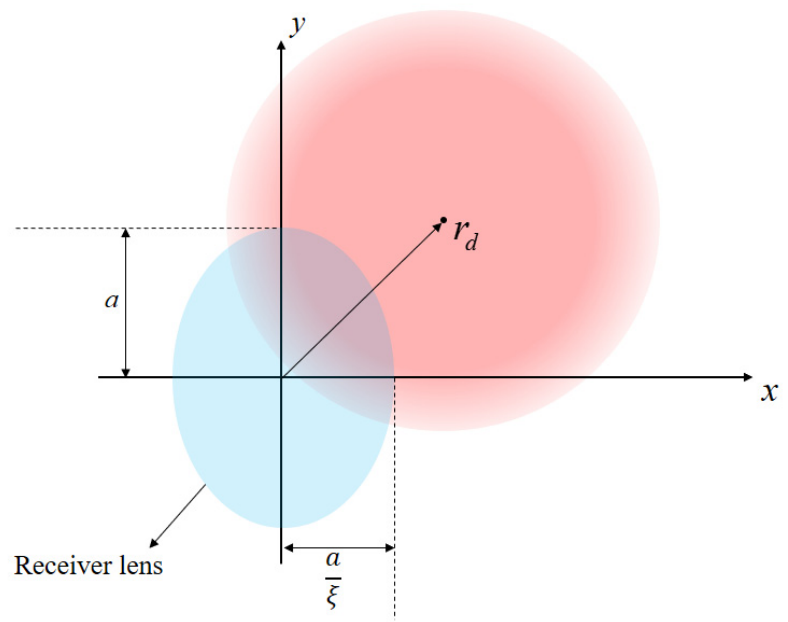

Figure 3. Geometry and pointing error diagram of the UAV optical communication in the flight state.

At a certain moment, suppose that the jitter induced by lateral wind resistance causes the lens plane to rotate by an angle $\alpha$ around the $y$ axis. The ellipse formed by the projection of the lens on the $x-y$ plane can be expressed as:

$$
\xi^{2} x^{2}+y^{2}=a^{2}
$$

where $\xi=1 / \cos \alpha$. The long axis of the ellipse is $a$, and the light intensity of the Gaussian beam section can be written as:

$$
I(r ; Z)=\frac{2}{\pi w_{z}^{2}} \exp \left(-\frac{2|r|^{2}}{w_{z}^{2}}\right)
$$

where $r$ is the radial vector with $r$ distance from the center of the light spot and $w_{z}$ is the beam waist at distance $Z$. The position deviation between the spot center and the lens $r_{d}$ is expressed as:

$$
r_{d}=r_{d_{r}}+r_{d_{t}}+Z \hat{\theta}_{t}
$$

where $\boldsymbol{r}_{d_{r}}=x_{r}+y_{r}$ is the position deviation of the receiving end, $\boldsymbol{r}_{d_{t}}=x_{t}+y_{t}$ is the position deviation of the transmitting end, $Z$ is the transmission distance, and $\hat{\theta}=\theta_{t x}+\theta_{t y}$ is the angle deviation of the transmitting end. Therefore, the geometric and pointing error coefficients can be expressed as:

$$
\begin{gathered}
h_{g}=\iint_{A} \frac{2}{\pi w_{z}^{2}} \exp \left[-2 \frac{\left(r+r_{d}\right)^{2}}{w_{z}^{2}}\right] d S \\
=\int_{-a}^{a} \int_{-\sqrt{\frac{a^{2}-y^{2}}{\xi^{2}}}}^{\sqrt{\frac{a^{2}-y^{2}}{\xi^{2}}}} \frac{2}{\pi w_{z}^{2}} \exp \left[-2 \frac{\left(x+x_{r}+x_{t}+Z \theta_{t x}\right)^{2}+\left(y+y_{r}+y_{t}+Z \theta_{t y}\right)^{2}}{w_{z}^{2}}\right] d y d x
\end{gathered}
$$

Equation (12) can be approximated as [20]: 


$$
h_{g} \simeq \frac{1}{\xi^{2}} A_{0} \exp \left(\frac{-2 r_{d}^{2}}{w_{z e q}^{2}}\right)
$$

where $A_{0}=[\operatorname{erf}(v)]^{2}$ is the ratio of the energy received by the lens to the total energy when $r_{d}=0$, and the equivalent beam waist can be expressed as:

$$
w_{z e q}^{2}=w_{z}^{2} \frac{\sqrt{\pi} \operatorname{erf}(v)}{2 \operatorname{vexp}\left(-v^{2}\right)}
$$

where $v=\sqrt{\pi} a / \sqrt{2} w_{z}$ and $\operatorname{erf}(\cdot)$ is the error function. According to the kinetic characteristic of the UAV, $\boldsymbol{r}_{d_{r}}, \boldsymbol{r}_{d_{t}}, \theta_{t}$, and $\alpha$ all obey Gaussian distributions with zero mean value. They can be expressed as $r_{d_{r}} \sim N\left(0, \sigma_{r p}^{2}\right), r_{d_{t}} \sim N\left(0, \sigma_{t p}^{2}\right), \theta_{t} \sim N\left(0, \sigma_{t o}^{2}\right)$, and $\alpha \sim N\left(0, \sigma_{\alpha}^{2}\right)$ respectively, where $\sigma_{t p}^{2}$ and $\sigma_{r p}^{2}$ are the variances of the position deviation of the UAV at the transmitting and receiving end, $\sigma_{t o}^{2}$ is the variance of the pointing angle deviation of the UAV at the transmitting end and $\sigma_{\alpha}^{2}$ is the variance of the jitter angle of the $\mathrm{UAV}$ at the receiving end caused by the horizontal lateral wind resistance. Accordingly, $\boldsymbol{r}_{d}$ conditioned on $\theta_{t x}$ and $\theta_{t y}$ has a Rician distribution, which can be expressed as:

$$
f\left(\boldsymbol{r}_{d} \mid \theta_{t}\right)=\frac{\boldsymbol{r}_{d}}{\sigma_{\text {to }}^{2}} \exp \left(-\frac{\boldsymbol{r}_{d}^{2}+\theta_{t}^{2} Z^{2}}{2 \sigma_{t o}^{2}}\right) I_{0}\left(\frac{\theta_{t} Z \boldsymbol{r}_{d}}{\sigma_{t o}^{2}}\right)
$$

where $I_{0}(\cdot)$ is the modified Bessel function of the first kind with order zero. The PDF of $\theta_{t}$ can be written as:

$$
f\left(\theta_{t}\right)=\frac{\theta_{t}}{\sigma_{t o}^{2}} \exp \left(-\frac{\theta_{t}{ }^{2}}{2 \sigma_{t o}^{2}}\right)
$$

According to Equations (13) and (15), the PDF of $h_{g}$ that conditioned on $\theta_{t}$ can be expressed as:

$$
f\left(h_{g} \mid \theta_{t}\right)=\frac{w_{z_{\text {eq }}}^{2}}{4 \xi^{2} A_{0} \sigma_{\text {to }}^{2}} \exp \left(-\frac{\theta_{t}^{2} Z^{2}}{2 \sigma_{\text {to }}^{2}}\right)\left(\frac{h_{g}}{A_{0}}\right)^{\frac{w_{\text {eqq }}^{2}}{4 \sigma_{\text {to }}^{2}}-1} \times I_{0}\left(\frac{\theta_{t} Z}{\sigma_{\text {to }}^{2}} \sqrt{-\frac{w_{z_{\text {eq }}}^{2}}{2} \ln \left(\frac{h_{g}}{A_{0}}\right)}\right)\left(0 \leq h_{g} \leq A_{0}\right)
$$

According to Equations (3), (8), and (17), the PDF of $h$ conditioned on $\theta_{t}$ can be expressed as:

$$
f\left(h \mid \theta_{t}\right)=\left\{1-\left[\frac{1}{2} \exp \left(-\frac{\left(\theta_{F O V}-\varphi\right)^{2}}{2\left(\sigma_{r_{0}}^{2}+\sigma_{t_{0}}^{2}\right)}\right)+\frac{1}{2} \exp \left(-\frac{\left(\theta_{F O V}+\varphi\right)^{2}}{2\left(\sigma_{r_{0}}^{2}+\sigma_{t_{0}}^{2}\right)}\right)\right]\right\} \times \int_{0}^{\infty} \frac{1}{h_{t}} f_{h_{g} \mid \theta_{t}}\left(h_{g}\right) f_{h_{t}}\left(h_{t}\right) d h_{t}
$$

Then the PDF of $h$ can be given as:

$$
f(h)=\int_{0}^{\infty} f_{h \mid \theta_{t}}(h) f_{\theta_{t}}\left(\theta_{t}\right) d \theta_{t}
$$

The outage probability of the UAV-FSO communication can be expressed as:

$$
P_{\text {out }}=\int_{0}^{h_{\text {th }}} f(h) \mathrm{d} h
$$

where $h_{t h}$ is the threshold of the channel coefficient $h$. When the instantaneous channel coefficient is lower than $h_{t h}$, the link outage can be considered.

Considering the modulation format of quadrature phase shift keying (QPSK), the average BER can be given by the following equation: 


$$
P_{e}=\frac{1}{2} \int_{0}^{\infty} \operatorname{erfc}\left(\sqrt{\frac{\gamma h}{2}}\right) f_{h}(h) d h
$$

where $\operatorname{erf} c(\cdot)$ is the complementary error function, $\gamma$ is the SNR at the transmitter. According to the above discussion, all of $h_{t}, h_{a}$, and $h_{g}$ can affect the signal intensity of the receiver, and further affect the SNR of the receiver, and ultimately the BER and the outage probability will be affected. In this paper, the influence of $h$ on the average BER and the outage probability is simulated by numerical Monte Carlo method.

\section{Performance Analysis of the In-Flight UAV FSO Communication Link}

The light carrier satisfies the Gaussian distribution. According to the previous contributions and the characteristics of the real UAV FSO communications, initial parameters are set as follows: the wavelength is $1550 \mathrm{~nm}$, the beam waist is $5 \mathrm{~mm}$, the radius of the receiving aperture is $0.1 \mathrm{~m}$, the transmission distance is $2 \mathrm{~km}$, and the turbulent outer scale $L_{0}$ and inner scales $l_{0}$ are $100 \mathrm{~m}$ and $0 \mathrm{~m}$, respectively. Modified Von Karman model is used to simulate the power spectral density function, in which the atmospheric refractive index structure parameter $C_{n}^{2}$ is $5 \times 10^{-15} \mathrm{~m}^{-2 / 3}$ and the transmission rate of the QSPK signal is $20 \mathrm{~Gb} / \mathrm{s}$. The simulation of system devices has been carried out using the AWGN model, and the simulation of the error caused by the modulation process has been carried out using the load random phase noise on the signal. The influence of atmospheric turbulence on the signal is loaded on the optical carrier by the phase screen method.

\subsection{Transmission Analysis of the FSO Communication for UAV in Straight Flight}

In this section, the simulation of the FSO communication process when the receivingend UAV is in the state of straight flight is carried out. The influence of the fuselage tilt angle of the UAV in horizontal and straight flights on the communication quality is analyzed, considering the angle pointing deviation and position deviation caused by the UAV jitter. In the simulation, setting $\theta_{F O V}=40 \mathrm{mrad}, \sigma_{r p}^{2}=\sigma_{t p}^{2}=1 \mathrm{~cm}, \sigma_{r o}^{2}=\sigma_{t o}^{2}=1 \mathrm{mrad}$, and select the tilt angles of the fuselage as $0,5 \mathrm{mrad}, 10 \mathrm{mrad}$, and $20 \mathrm{mrad}$, respectively.

Figure 4 shows the distribution of the optical communication channel coefficient under different fuselage tilt angles when the UAV flies in a straight line on the same horizontal plane. As the tilt angle of the fuselage increases, the value of the channel coefficient $h$ gradually becomes smaller. Therefore, the FSO communication quality between UAVs has also deteriorated.

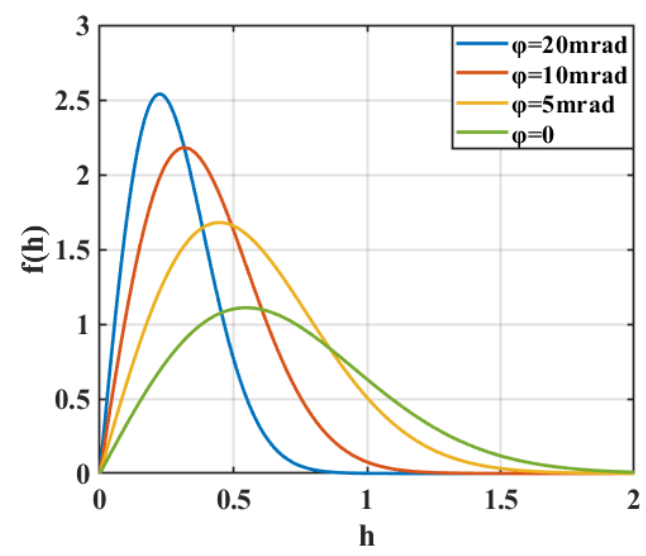

Figure 4. Normalized distribution of the channel coefficient at different tilt angles when the UAV flies straight at the same altitude.

As shown in Figure 5, when the UAV flies in a straight line on the same horizontal plane, the fuselage tilt will greatly influence the communication quality. As shown in Figure $5 \mathrm{a}$, the outage probability of the UAV optical communication link increases as the 
tilt angle increases. When $\varphi=0$, that is the states of UAV are similar to that with hovering, the outage probability is close to $10^{-4}$ when the SNR is $20 \mathrm{~dB}$. However, increasing the tilt angle of the UAV to $20 \mathrm{mrad}$, the outage probability becomes greater than $10^{-2}$ under the same SNR. In order to make the outage probability reach $10^{-3}$, the SNR should be increased from $16 \mathrm{~dB}$ to more than $20 \mathrm{~dB}$ when the tilt angle of the UAVs increases from 0 to 10 mrad.

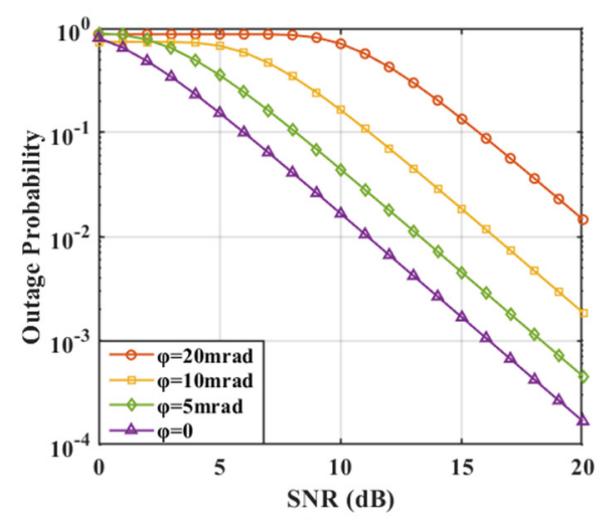

(a)

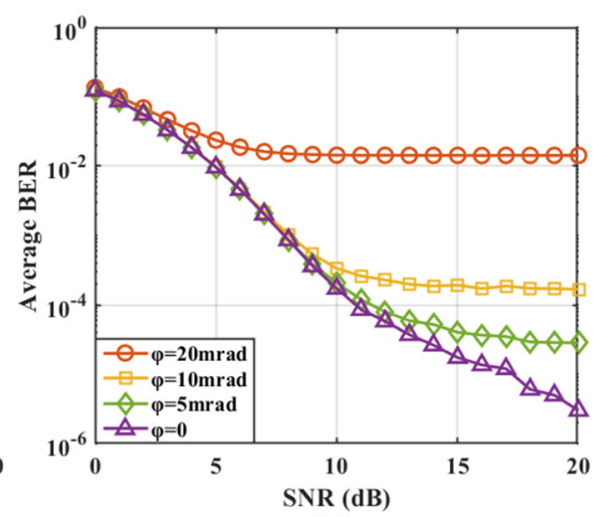

(b)

Figure 5. FSO communication performance of UAVs at different tilt angles when the UAV flies straightly at the same altitude. (a) Outage probability; (b) average BER.

As shown in Figure 5b, the BER will increase as the tilt angle of the UAV increases. For example, when the SNR equals $20 \mathrm{~dB}$, the BER will increase from $2 \times 10^{-6}$ to $10^{-2}$ when the tilt angle of the UAV increases from 0 to $20 \mathrm{mrad}$. To make the average BER reach $10^{-5}$, the SNR should be raised from $13 \mathrm{~dB}$ to $20 \mathrm{~dB}$ when the tilt angle of the UAVs increases from 0 to $5 \mathrm{mrad}$. When the tilt angle is limited (less than $10 \mathrm{mrad}$ ), the UAV can achieve reliable communication just by increasing the SNR. However, when the tilt angle continues to increase, the communication quality will be seriously disturbed. When the tilt angle is greater than $10 \mathrm{mrad}$, the average BER is still higher than $10^{-4}$ even if the SNR reaches $20 \mathrm{~dB}$. When the tilt angle is too large, only a small part of the light can reach the detection area after passing through the lens, and the caused communication interruption and symbol loss seriously limit the upper limit of the performance of the receiving system. Therefore, even if the SNR increases, the BER does not decrease significantly. As a result, the optical communication link between UAVs cannot be established at this time.

\subsection{Transmission Analysis of the FSO Communication for UAV during Yaw Flight}

The optical communication process of the receiving-end UAV in the state of yawing flight is simulated by setting $\theta_{F O V}=40 \mathrm{mrad}, \sigma_{r p}^{2}=\sigma_{t p}^{2}=1 \mathrm{~cm}, \sigma_{r o}^{2}=\sigma_{t o}^{2}=1 \mathrm{mrad}$. However, the fuselage still has an angle to the $y$ axis while the UAV is yawing. Therefore, this simulation sets the tilt angle $\varphi$ as $5 \mathrm{mrad}$, and the variance of the random angle deflection around the $y$ axis $\sigma_{\alpha}^{2}=0 \mathrm{mrad}, 1 \mathrm{mrad}, 2 \mathrm{mrad}$, and $5 \mathrm{mrad}$, respectively.

Figure 6 shows the distribution of the FSO communication channel coefficient under different fuselage tilt angles when the UAV flies in yaw motion on the same horizontal plane. As the wind resistance increases, the swing range of the UAV around the $y$ axis becomes larger. The value of the UAV FSO communication channel coefficient $h$ gradually becomes smaller, which means that the quality of the optical communication is getting worse.

As can be seen from Figure 7, the influence of UAVs' jitter caused by the wind resistance on its communication quality is less than that of the tilt angle of the fuselage. In Figure $7 \mathrm{a}$, with the increase of the random wind resistance, the outage probability of the UAVs' optical communication slightly increases. Under the condition that the SNR is equal to $20 \mathrm{~dB}$, the outage probability of the link increases to $10^{-3}$ when $\sigma_{\alpha}^{2}$ increases from 0 to 5 mrad. With the requirement of the outage probability reaching $10^{-3}$, the SNR should be increased from $17 \mathrm{~dB}$ to more than $20 \mathrm{~dB}$ when $\sigma_{\alpha}^{2}$ increases from 0 to 5 mrad. 


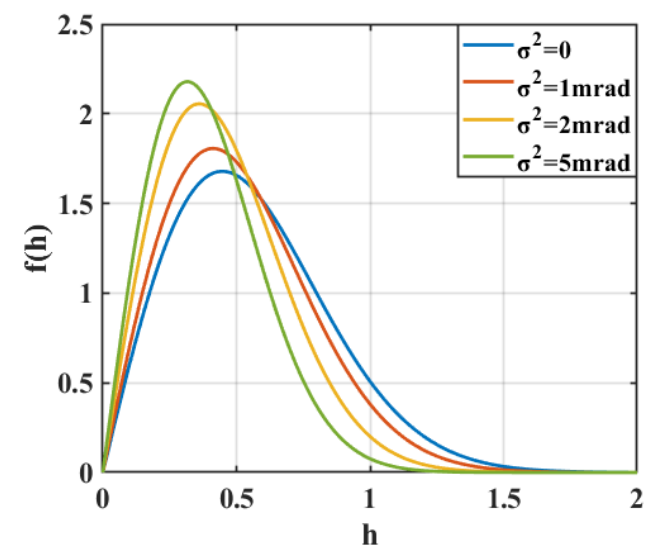

Figure 6. Normalized distribution of the channel coefficient with different wind resistances in yaw motion.

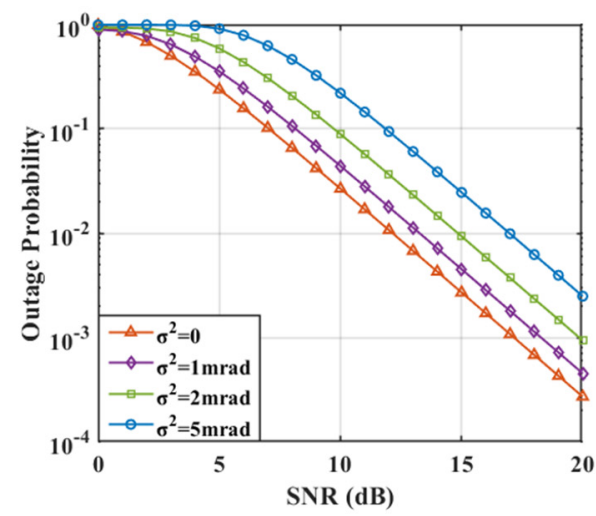

(a)

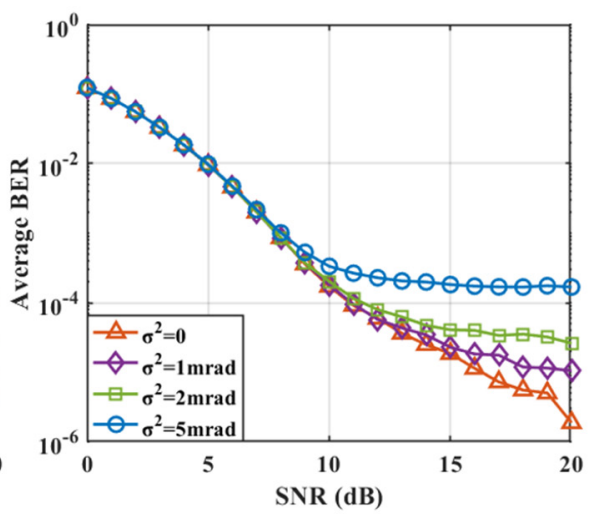

(b)

Figure 7. FSO communication performance with different wind resistances of the UAV in yaw motion at the same horizontal plane. (a) Outage probability; (b) average BER.

In Figure $7 \mathrm{~b}$, the BER will also increase when $\sigma_{\alpha}^{2}$ increases. At the SNR of $20 \mathrm{~dB}$, the BER will increase from $3 \times 10^{-6}$ to $2 \times 10^{-4}$ when $\sigma_{\alpha}^{2}$ increases from 0 to 5 mrad. The effect of the horizontal random wind resistance on the UAV FSO communication is smaller than the influence of the tilt angle. With the requirement of the average BER reaching $10^{-5}$, the SNR should be $15 \mathrm{~dB}, 17 \mathrm{~dB}$, and $20 \mathrm{~dB}$ when $\sigma_{\alpha}^{2}$ is $0 \mathrm{mrad}, 1 \mathrm{mrad}$, and $2 \mathrm{mrad}$, respectively. However, when $\sigma_{\alpha}^{2}$ is $5 \mathrm{mrad}$, the average BER is still higher than $10^{-4}$ even if the SNR reaches $20 \mathrm{~dB}$. In this case, it is considered that the communication quality between UAVs is slightly lower than the critical point of effective communication. Although communication can be established, the link is relatively unstable.

\section{In-Flight UAV FSO Communication Improvement Based on Spatial Diversity}

The spatial diversity technology can effectively reduce the influence of the phase distortion and light intensity fluctuation, so as to achieve the signal gain [13]. In the UAV FSO link, the spatial diversity with single-in and multiple-out (SIMO) is suitable to be adopted, as shown in Figure 8, in which each optical antenna in the receiving array independently receives the optical signals. After coupling to the fiber, the signal will be compensated and reconstructed by their corresponding receiver, and the equal gain merge (EGC) algorithm will be used to merge each signal [21]. 


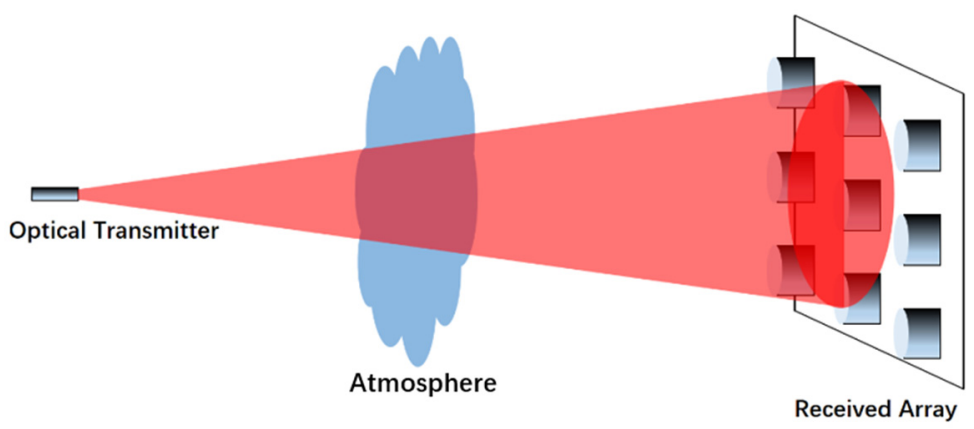

Figure 8. Schematic diagram of the spatial diversity receiving structure for SIMO FSO communications.

The linear combining process for the diversity technology can be expressed as:

$$
y=\sum_{i=1}^{M} \widetilde{w}_{i}(t) x_{i}(t)
$$

where $x_{i}(t)$ is the signal of each path, which can be expressed as:

$$
x_{i}(t)=a_{i}(t)+n_{i}(t)
$$

where $a_{i}(t)$ denotes the signal, and $n_{i}(t)$ is the noise. $\widetilde{w}_{i}(t)$ represents the weight coefficient of each signal in the process of merging, which can be expressed as:

$$
\widetilde{w}_{i}(t)=w_{i}(t) \exp \left[j \theta_{i}(t)\right]
$$

where the real part $w_{i}(t)$ represents the proportion of signals in each path, and the imaginary part $e^{j \theta_{i}(t)}$ represents the phase alignment of signals in each path to achieve coherent combining effect. Equal-gain combining (EGC) algorithm simplifies all the weighting coefficients to 1 and only performs phase alignment. It can both effectively improve the SNR of the combined signal and avoid the contingency of results.

In this simulation, two and four receivers are considered respectively for comparison. The parameters are shown as follows: the field angle $\theta_{F O V}=40 \mathrm{mrad}$, the variance of transceiver end position deviation $\sigma_{r p}^{2}=\sigma_{t p}^{2}=1 \mathrm{~cm}$, the variances of the angular deviation $\sigma_{r o}^{2}=\sigma_{t o}^{2}=1 \mathrm{mrad}$, the fuselage of the tilt angle $\varphi=5 \mathrm{mrad}$, and the random angle deflection variance around the $y$ axis $\sigma_{\alpha}^{2}=1 \mathrm{mrad}$. The number of receivers in the receiving array is 1,2, and 4, respectively. Figure 9 shows the BER performance against the SNR, which represents the quality of the FSO communication link.

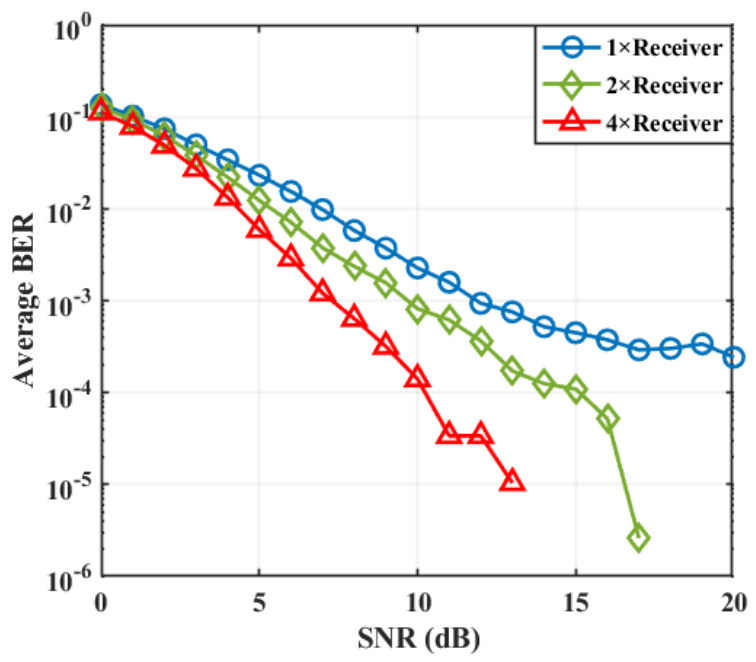

Figure 9. FSO communication performance of the UAV with single-, double- and four-receiver arrays. 
As shown in Figure 9, the average BER is significantly reduced compared to that without the diversity receiving technology, and the transmission performance of the UAV FSO system is effectively improved. Without the diversity receiving technology, the average BER of the received signal is still higher than $10^{-4}$ under the SNR higher than $20 \mathrm{~dB}$, and the FSO communication link of the UAV is unstable. When the receiving array composed of two receivers is used, the average BER is less than $10^{-4}$ when the SNR is higher than $15 \mathrm{~dB}$. When the receiving array composed of four receivers is used, the average BER is less than $10^{-4}$ when the SNR is higher than $10 \mathrm{~dB}$. When the SNR is around $13 \mathrm{~dB}$, the average BER drops to $10^{-5}$. Therefore, it can be seen that the application of diversity receiving technology can effectively improve the quality of communication and enhance the stability of the link.

\section{Conclusions}

An in-flight UAV FSO communication channel model has been established. In addition to the atmospheric turbulence and UAV's jitter caused by its motor vibration, the tilt angle and the horizontal lateral wind resistance caused by UAV's different motion states have also been considered. Besides, the results of FSO communication processes under different flight states (hover state, flight state, and static state) have been compared. Analysis results show that when the BER is required to reach $10^{-5}$, the SNR should be increased from $13 \mathrm{~dB}$ to $20 \mathrm{~dB}$ when the tilt angle of the UAVs increases from 0 to $5 \mathrm{mrad}$. With the requirement of the average BER reaching $10^{-5}$, the SNR should be $15 \mathrm{~dB}, 17 \mathrm{~dB}$, and $20 \mathrm{~dB}$ when the variance of the wind $\sigma_{\alpha}^{2}$ is 0,1 , and $2 \mathrm{mrad}$, respectively. It is verified that the spatial diversity receiving technology can effectively improve the communication quality between UAVs. If the spatial diversity array with four receivers is used, the BER of lower than $10^{-5}$ can be obtained just with a SNR of $13 \mathrm{~dB}$.

Author Contributions: A.Z. contributed to the paper in methodology, program, visualization, data analysis and paper draft. Y.H. contributed to the paper in investigation, resources and project administration. S.G. contributed to the paper in conceptualization, supervision, review and editing. All authors have read and agreed to the published version of the manuscript.

Funding: This research was funded by the National Natural Science Foundation of China (61875172), the Zhejiang Provincial Natural Science Foundation of China (LD19F050001), and the Fundamental Research Funds for the Central Universities (2020XZZX005-07), and the National Key Research and Development Program of China (2019YFB2205202).

Institutional Review Board Statement: Not applicable.

Informed Consent Statement: Not applicable.

Data Availability Statement: Not applicable.

Acknowledgments: The author acknowledges the valuable comments of the reviewers.

Conflicts of Interest: The authors declare no conflict of interest.

\section{References}

1. Alzenad, M.; Shakir, M.Z.; Yanikomeroglu, H.; Alouini, M.-S. FSO-Based vertical backhaul/fronthaul framework for 5G+wireless networks. IEEE Commun. Mag. 2018, 56, 218-224. [CrossRef]

2. Ghassemlooy, Z.; Popoola, W.; Rajbhandari, S. Optical Wireless Communications, 1st ed.; CRC Press: Foca Raton, FL, USA, 2017.

3. Khalighi, M.A.; Uysal, M. Survey on free space optical communication: A communication theory perspective. IEEE Commun. Surv. Tutor. 2014, 16, 2231-2258. [CrossRef]

4. Michailidis, E.T.; Nomikos, N.; Bithas, P.; Vouyioukas, D.; Kanatas, A.G. Outage probability of triple-hop mixed RF/FSO/RF stratospheric communication systems. In Proceedings of the 10th International Conference on Advances in Satellite and Space Communications (SPACOMM 2018), Athens, Greece, 22-26 April 2018; pp. 1-6.

5. Fawaz, W.; Abou-Rjeily, C.; Assi, C. UAV-Aided cooperation for FSO communication systems. IEEE Commun. Mag. 2018, 56, 70-75. [CrossRef]

6. Cruz, P.J.; Fierro, R. Towards optical wireless communications between micro unmanned aerial and ground Systems. In Proceedings of the 2015 International Conference on Unmanned Aircraft Systems, Denver, CO, USA, 9-12 June 2015; pp. 669-676. 
7. Dautov, K.; Arzykulov, S.; Nauryzbayev, G.; Kizilirmak, R.C. On the performance of UAV-enabled multihop V2V FSO systems over generalized alpha - $\mu$ channels. In Proceedings of the 2018 International Conference on Computing and Network Communications (CoCoNet), Astana, Kazakhstan, 15-17 August 2018; pp. 79-83.

8. Li, L.; Zhang, R.; Liao, P.; Cao, Y.; Song, H.; Zhao, Y.; Du, J.; Zhao, Z.; Liu, C.; Pang, K.; et al. Mitigation for turbulence effects in a 40-Gbit/s orbital-angular-momentum-multiplexed free-space optical link between a ground station and a retro-reflecting UAV using MIMO equalization. Opt. Lett. 2019, 44, 5181-5184. [CrossRef] [PubMed]

9. Dabiri, M.T.; Sadough, M.S.; Khalighi, M.A. Channel modeling and parameter optimization for hovering UAV-Based free-space optical links. IEEE J. Sel. Areas Commun. 2018, 36, 2104-2113. [CrossRef]

10. Dabiri, M.T.; Sadough, S.M.S.; Ansari, I.S. Tractable optical channel modeling between UAVs. IEEE Trans. Veh. Technol. 2019, 68, 11543-11550. [CrossRef]

11. Najafi, M.; Ajam, H.; Jamali, V.; Diamantoulakis, P.D.; Karagiannidis, G.K.; Schober, R. Statistical Modeling of the FSO Fronthaul Channel for UAV-Based Communications. IEEE Trans. Commun. 2020, 68, 3720-3736. [CrossRef]

12. Chlestil, C.; Leitgeb, E.; Schmitt, N.P.; Muhammad, S.S.; Zettl, K.; Rehm, W. Reliable optical wireless links within UAV swarms. In Proceedings of the 2006 International Conference on Transparent Optical Networks, Nothingam, UK, 18-22 June 2006 ; p. 39.

13. Ibrahim, M.M.; Ibrahim, A.M. Performance analysis of optical receivers with space diversity reception. IEE Proc. Commun. 1996, 143, 369-372. [CrossRef]

14. Karagiannidis, G.K.; Zogas, D.A.; Sagias, N.C.; Kotsopoulos, S.A.; Tombras, G.S. Equal-gain and maximal-ratio combining over nonidentical Weibull fading channels. IEEE Trans. Wirel. Commun. 2005, 4, 841-846. [CrossRef]

15. Li, K.; Ma, J.; Belmonte, A.; Tan, L.; Yu, S. Performance analysis of satellite-to-ground downlink optical communications with spatial diversity over gamma-gamma atmospheric turbulence. Opt. Eng. 2015, 54, 126103. [CrossRef]

16. Al-Habash, M.A.; Al-Habash, M.A.; Andrews, L.C.; Andrews, L.C.; Phillips, R.L. Mathematical model for the irradiance probability density function of a laser beam propagating through turbulent media. Opt. Eng. 2001, 40, 1554-1562. [CrossRef]

17. Whitfield, E.M.; Banerjee, P.P.; Haus, J.W. Propagation of Gaussian beams through a modified von Karman phase screen. In Proceedings of the SPIE Optical Engineering + Applications, San Diego, CA, USA, 12-16 August 2012; Volume 8517.

18. Chatterjee, M.R.; Mohamed, F.H.A. Investigation of profiled beam propagation through a turbulent layer and temporal statistics of diffracted output for a modified von Karman phase screen. In Proceedings of the SPIE LASE, San Francisco, CA, USA, 1-6 February 2014; Volume 897102.

19. Gudimetla, V.S.R.; Holmes, R.B.; Farrell, T.C.; Lucas, J. Phase screen simulations of laser propagation through non-Kolmogorov atmospheric turbulence. In Proceedings of the SPIE Defense, Security and Sensing, Orlando, FL, USA, 25-29 April 2011; Volume 803808 .

20. Farid, A.A.; Hranilovic, S. Outage capacity optimization for free-space optical links with pointing errors. J. Lightwave Technol. 2007, 25, 1702-1710. [CrossRef]

21. Yu, Z.; Cai, R.; Wu, Z.; He, H.; Jiang, H.; Feng, X.; Zheng, A.; Chen, J.; Gao, S. Performance evaluation of direct-detection coherent receiver array for free-space communications with full-link simulation. Opt. Commun. 2020, 454. [CrossRef] 